\title{
Existence and exponential stability of periodic solutions for a class of Hamiltonian systems on time scales
}

\author{
Li Yang ${ }^{1}$, Yongzhi Liao² and Yongkun Li ${ }^{1 *}$
}

"Correspondence: yklie@ynu.edu.cn 1 Department of Mathematics, Yunnan University, Kunming, Yunnan 650091, People's Republic of China

Full list of author information is available at the end of the article

\begin{abstract}
In this paper, by using a fixed point theorem and the theory of calculus on time scales, we obtain some sufficient conditions for the existence and exponential stability of periodic solutions for a class of Hamiltonian systems on time scales. We also present numerical examples to show the feasibility of our results. The results of this paper are completely new and complementary to the previously known results even if the time scale $\mathbb{T}=\mathbb{R}$ or $\mathbb{Z}$.
\end{abstract}

Keywords: periodic solution; Hamiltonian system; exponential stability; time scale

\section{Introduction}

Hamiltonian system, which was introduced by the Irish mathematician SWR Hamilton, is widely used in mathematical sciences, life sciences and so on. Many models in celestial mechanics, plasma physics, space science and bio-engineering are in the form of a Hamilton system. Therefore, the study of a Hamilton system is useful and meaningful in theory and practice. Recently, various Hamiltonian systems have been extensively studied (see [1-5] and references cited therein).

Most existing results on the study of Hamiltonian systems are for continuous systems. However, both discrete and continuous systems are important in applications, and it is troublesome to study the dynamical properties for continuous and discrete systems, respectively. Therefore, it is meaningful to study dynamical systems on time scales (see [613] and references cited therein), which helps avoid proving results twice, once for differential equations and once for difference equations. There have been some results devoted to Hamiltonian systems on time scales [9, 10, 14-17]. For example, authors in [14] introduced Hamiltonian systems on time scales and authors in $[15,16]$ studied the following Hamiltonian system on time scales:

$$
\left\{\begin{array}{l}
x^{\Delta}(t)=\alpha(t) x(\sigma(t))+\beta(t) y(t), \\
y^{\Delta}(t)=-\gamma(t) x(\sigma(t))-\alpha(t) y(t),
\end{array}\right.
$$

where $t \in \mathbb{T}$. In [15], authors obtained inequalities of Lyapunov for (1.1), and authors in [16] studied the stability of (1.1) by using Floquet theory. However, to the best of our knowledge, up to now, there have been no papers published on the existence and exponential stability of a periodic solution to (1.1).

@ 2013 Yang et al.; licensee Springer. This is an Open Access article distributed under the terms of the Creative Commons Attribution License (http://creativecommons.org/licenses/by/2.0), which permits unrestricted use, distribution, and reproduction in any medium, provided the original work is properly cited. 
Motivated by the above mentioned works, in this paper, we study the existence and exponential stability of periodic solutions to (1.1), in which $\mathbb{T}$ is a periodic time scale. The main aim of this paper is to study the existence of periodic solutions to (1.1) by using a fixed point theorem. Moreover, we also study the exponential stability of the periodic solution to (1.1). Our results are new and complementary to the previously known results even if the time scale $\mathbb{T}=\mathbb{R}$ or $\mathbb{Z}$.

Remark 1.1 It is obvious that when $\mathbb{T}=\mathbb{R},(1.1)$ reduces to the following continuous time Hamiltonian system:

$$
x^{\prime}(t)=\alpha(t) x(t)+\beta(t) y(t), \quad y^{\prime}(t)=-\gamma(t) x(t)-\alpha(t) y(t), \quad t \in \mathbb{R} .
$$

When $\mathbb{T}=\mathbb{Z}$, (1.1) reduces to the following discrete time Hamiltonian system:

$$
\Delta x(n)=\alpha(n) x(n+1)+\beta(n) y(n), \quad \Delta y(n)=-\gamma(n) x(n+1)-\alpha(n) y(n), \quad n \in \mathbb{Z} .
$$

For convenience, we denote $[a, b]_{\mathbb{T}}=\{t \mid t \in[a, b] \cap \mathbb{T}\}$ and $\vartheta=\sup _{t \in \mathbb{T}} \mu(t)$. For an $\omega$ periodic function $f: \mathbb{T} \rightarrow \mathbb{R}$, we denote $f^{+}=\max _{t \in[0, \omega]_{\mathbb{T}}}|f(t)|, f^{-}=\min _{t \in[0, \omega]_{\mathbb{T}}}|f(t)|$ and $\bar{f}=\max _{t \in[0, \omega]_{\mathbb{T}}} f(t)$.

Throughout this paper, we assume that

$\left(\mathrm{H}_{1}\right) \beta(t), \gamma(t) \in C_{r d}(\mathbb{T}, \mathbb{R}), \alpha(t) \in C_{r d}(\mathbb{T},(0,+\infty))$ are all $\omega$-periodic functions and $e_{-\alpha}(\omega$, $0) \neq 1,-\alpha \in \mathcal{R}^{+}$, where $\mathcal{R}^{+}=\mathcal{R}^{+}(\mathbb{T}, \mathbb{R})=\{r: 1+\mu(t) r(t)>0, \forall t \in \mathbb{T}\}$.

\section{Preliminaries}

In this section, we introduce some definitions and state some preliminary results.

Definition 2.1 [6] Let $\mathbb{T}$ be a nonempty closed subset (time scale) of $\mathbb{R}$. The forward and backward jump operators $\sigma, \rho: \mathbb{T} \rightarrow \mathbb{T}$ and the graininess $\mu: \mathbb{T} \rightarrow \mathbb{R}^{+}$are defined, respectively, by

$$
\sigma(t)=\inf \{s \in \mathbb{T}: s>t\}, \quad \rho(t)=\sup \{s \in \mathbb{T}: s<t\} \quad \text { and } \quad \mu(t)=\sigma(t)-t .
$$

Definition 2.2 [6] A point $t \in \mathbb{T}$ is called left-dense if $t>\inf \mathbb{T}$ and $\rho(t)=t$, left-scattered if $\rho(t)<t$, right-dense if $t<\sup \mathbb{T}$ and $\sigma(t)=t$, and right-scattered if $\sigma(t)>t$. If $\mathbb{T}$ has a left-scattered maximum $m$, then $\mathbb{T}^{k}=\mathbb{T} \backslash\{m\}$; otherwise $\mathbb{T}^{k}=\mathbb{T}$. If $\mathbb{T}$ has a right-scattered minimum $m$, then $\mathbb{T}^{k}=\mathbb{T} \backslash\{m\}$; otherwise $\mathbb{T}^{k}=\mathbb{T}$.

Definition 2.3 [6] A function $r: \mathbb{T} \rightarrow \mathbb{R}$ is called regressive if

$$
1+\mu(t) r(t) \neq 0
$$

for all $t \in \mathbb{T}^{k}$. If $r$ is a regressive function, then the generalized exponential function $e_{r}$ is defined by

$$
e_{r}(t, s)=\exp \left\{\int_{s}^{t} \xi_{\mu(\tau)}(r(\tau)) \Delta \tau\right\} \quad \text { for } s, t \in \mathbb{T}
$$


with the cylinder transformation

$$
\xi_{h}(z)= \begin{cases}\frac{\log (1+h z)}{h} & \text { if } h \neq 0 \\ z & \text { if } h=0\end{cases}
$$

Let $p, q: \mathbb{T} \rightarrow \mathbb{R}$ be two regressive functions, we define

$$
p \oplus q:=p+q+\mu p q, \quad \ominus p:=-\frac{p}{1+\mu p}, \quad p \ominus q:=p \oplus(\ominus q) .
$$

Then the generalized exponential function has the following properties.

Lemma 2.1 [6] Assume that $p, q: \mathbb{T} \rightarrow \mathbb{R}$ are two regressive functions, then

(i) $e_{0}(t, s) \equiv 1$ and $e_{p}(t, t) \equiv 1$

(ii) $e_{p}(\sigma(t), s)=(1+\mu(t) p(t)) e_{p}(t, s)$;

(iii) $e_{p}(t, s)=\frac{1}{e_{p}(s, t)}=e_{\ominus p}(s, t)$;

(iv) $e_{p}(t, s) e_{p}(s, r)=e_{p}(t, r)$.

Lemma 2.2 [7] Assume that $f, g: \mathbb{T} \rightarrow \mathbb{R}$ are delta differentiable at $t \in \mathbb{T}^{k}$, then

(i) $\left(v_{1} f+v_{2} g\right)^{\Delta}=v_{1} f^{\Delta}+v_{2} g^{\Delta}$ for any constants $v_{1}, v_{2}$;

(ii) $(f g)^{\Delta}(t)=f^{\Delta}(t) g(t)+f(\sigma(t)) g^{\Delta}(t)=f(t) g^{\Delta}(t)+f^{\Delta}(t) g(\sigma(t))$.

Lemma 2.3 [7] Assume that $p(t) \geq 0$ for $t \geq s$, then $e_{p}(t, s) \geq 1$.

Definition 2.4 [7] A function $f: \mathbb{T} \rightarrow \mathbb{R}$ is positively regressive if $1+\mu(t) f(t)>0$ for all $t \in \mathbb{T}$.

Lemma 2.4 [7] Suppose that $p \in \mathcal{R}^{+}$, then

(i) $e_{p}(t, s)>0$ for all $t, s \in \mathbb{T}$;

(ii) if $p(t) \leq q(t)$ then $e_{p}(t, s) \leq e_{q}(t, s)$ for $t, s \in \mathbb{T}$.

Lemma 2.5 [18] If $p \in \mathcal{R}^{+}$and $p(t)<0$ for all $t \in \mathbb{T}$, then for all $s \in \mathbb{T}$ with $s \leq t$, we have

$$
0<e_{p}(t, s) \leq \exp \left(\int_{s}^{t} p(u) \Delta u\right)<1
$$

Lemma 2.6 [8] Let $x \in\{x \in C(\mathbb{T}, \mathbb{R}) \mid x(t+\omega)=x(t)\}$. Then $\left\|x^{\sigma}\right\|$ exists and $\left\|x^{\sigma}\right\|=\|x\|$, where $\|x\|=\max _{t \in[0, \omega]_{\mathbb{T}}}|x(t)|$.

Lemma 2.7 [7] If $p \in \mathcal{R}$ and $a, b, c \in \mathbb{T}$, then

$$
\left[e_{p}(c, \cdot)\right]^{\Delta}=-p\left[e_{p}(c, \cdot)\right]^{\sigma}
$$

and

$$
\int_{a}^{b} p(t) e_{p}(c, \sigma(t)) \Delta t=e_{p}(c, a)-e_{p}(c, b)
$$


Definition 2.5 Let $z^{*}(t)=\left(x^{*}(t), y^{*}(t)\right)^{T}$ be a solution of (1.1) and $z(t)=(x(t), y(t))^{T}$ be an arbitrary solution of (1.1). If there exist positive constants $M>1$ and $\lambda$ with $\ominus \lambda \in \mathcal{R}^{+}$such that for $t_{0} \in \mathbb{T}$,

$$
\left|z(t)-z^{*}(t)\right|_{1} \leq M\left\|z-z^{*}\right\|_{1} e_{\ominus \lambda}\left(t, t_{0}\right), \quad t \in \mathbb{T}, t \geq t_{0},
$$

where $|z(t)|_{1}=\max \left\{\left|x(t)-x^{*}(t)\right|,\left|y(t)-y^{*}(t)\right|\right\},\left\|z-z^{*}\right\|_{1}=\max \left\{\left|z_{1}-z_{1}^{*}\right|_{0},\left|z_{2}-z_{2}^{*}\right|_{0}\right\}, \mid z_{i}-$ $\left.z_{i}^{*}\right|_{0}=\max _{s \in\left(-\infty, t_{0}\right]_{\mathbb{T}}}\left|z_{i}(s)-z_{i}^{*}(s)\right|, i=1,2$, then the solution $z^{*}(t)$ is said to be exponentially stable.

\section{Existence and uniqueness}

Lemma 3.1 Let $\left(\mathrm{H}_{1}\right)$ hold. Then every $\omega$-periodic solution $(x(t), y(t))^{T}$ of $(1.1)$ is of the form:

$$
\left\{\begin{array}{l}
x(t)=\frac{1}{e_{-\alpha}(\omega, 0)-1} \int_{t}^{t+\omega} \beta(s) y(s) e_{-\alpha}(s, t) \Delta s, \\
y(t)=\frac{1}{1-e_{\ominus}(-\alpha)(\omega, 0)} \int_{t}^{t+\omega} \frac{e_{\ominus}(-\alpha)(s, t)}{1-\mu(s) \alpha(s)} \gamma(s) x(\sigma(s)) \Delta s .
\end{array}\right.
$$

Proof Let $(x(t), y(t))^{T}$ be an $\omega$-periodic solution of (1.1). We can rewrite the first equation of (1.1) as follows:

$$
x^{\Delta}(t)-\alpha(t) x(\sigma(t))=\beta(t) y(t) .
$$

Multiplying both sides of the above equation by $e_{-\alpha}(t, 0)$, we have

$$
\left(e_{-\alpha}(t, 0) x(t)\right)^{\Delta}=e_{-\alpha}(t, 0) \beta(t) y(t) .
$$

Integrating both sides of this equation from $t$ to $t+\omega$ and noticing that $x(t+\omega)=x(t)$, we have

$$
x(t)=\frac{1}{e_{-\alpha}(\omega, 0)-1} \int_{t}^{t+\omega} \beta(s) y(s) e_{-\alpha}(s, t) \Delta s .
$$

On the other hand, we rewrite the second equation of (1.1) as follows:

$$
(1-\alpha(t) \mu(t)) y^{\Delta}(t)+\alpha(t) y(\sigma(t))=-\gamma(t) x(\sigma(t))
$$

which is equivalent to

$$
y^{\Delta}(t)+\ominus(-\alpha(t)) y(\sigma(t))=-\frac{\gamma(t)}{1-\alpha(t) \mu(t)} x(\sigma(t)) .
$$

Multiplying both sides of the above equation by $e_{\ominus(-\alpha)}(t, 0)$, we have

$$
\left(e_{\ominus(-\alpha)}(t, 0) y(t)\right)^{\Delta}=-\gamma(t) x(\sigma(t)) e_{\ominus(-\alpha)}(\sigma(t), 0) .
$$

Integrating both sides of this equation from $t$ to $t+\omega$ and noticing that $y(t+\omega)=y(t)$, we have

$$
y(t)=\frac{1}{1-e_{\ominus(-\alpha)}(\omega, 0)} \int_{t}^{t+\omega} \frac{e_{\ominus(-\alpha)}(s, t)}{1-\mu(s) \alpha(s)} \gamma(s) x(\sigma(s)) \Delta s,
$$

which completes the proof. 
Theorem 3.1 Assume that $\left(\mathrm{H}_{1}\right)$ and

$\left(\mathrm{H}_{2}\right) \min _{s \in[0, \omega]_{\mathbb{T}}}\{|1-\mu(s) \alpha(s)|\} \neq 0$ and

$$
\theta=\max \left\{\frac{\beta^{+} \omega e^{\int_{0}^{\omega}\left|\xi_{\mu(\tau)}(-\alpha(\tau))\right| \Delta \tau}}{\left|e_{-\alpha}(\omega, 0)-1\right|}, \frac{\gamma^{+} \omega e^{\int_{0}^{\omega}\left|\xi_{\mu(\tau)}(-\alpha(\tau))\right| \Delta \tau}}{\left|1-e_{\ominus(-\alpha)}(\omega, 0)\right| \min _{s \in[0, \omega]_{\mathbb{T}}}\{1-\mu(s) \alpha(s) \mid\}}\right\}<1
$$

hold. Then (1.1) has a unique periodic solution.

Proof Let $\mathbb{X}=\left\{z(t)=(x(t), y(t))^{T} \in C\left(\mathbb{T}, \mathbb{R}^{2}\right) \mid x(t+\omega)=x(t), y(t+\omega)=y(t)\right\}$ with the norm $\|z\|=\max \left\{|x|_{0},|y|_{0}\right\}$, where $|x|_{0}=\max _{t \in[0, \omega]_{\mathbb{T}}}|x(t)|$ and $|y|_{0}=\max _{t \in[0, \omega]_{\mathbb{T}}}|y(t)|$. Then $\mathbb{X}$ is a Banach space. For $z \in \mathbb{X}$, define the following operator:

$$
\Phi: \mathbb{X} \rightarrow \mathbb{X}, \quad z=(x, y)^{T} \rightarrow \Phi z=\left(\Phi_{1} z, \Phi_{2} z\right)^{T},
$$

where

$$
\Phi_{1} z(t)=\frac{1}{e_{-\alpha}(\omega, 0)-1} \int_{t}^{t+\omega} \beta(s) y(s) e_{-\alpha}(s, t) \Delta s
$$

and

$$
\Phi_{2} z(t)=\frac{1}{1-e_{\ominus(-\alpha)}(\omega, 0)} \int_{t}^{t+\omega} \frac{e_{\ominus(-\alpha)}(s, t)}{1-\mu(s) \alpha(s)} \gamma(s) x(\sigma(s)) \Delta s .
$$

We will show that $\Phi$ is a contraction. First we show that for any $z \in \mathbb{X}$, we have $\Phi z \in \mathbb{X}$. Note that

$$
\begin{aligned}
\Phi_{1} z(t+\omega) & =\frac{1}{e_{-\alpha}(\omega, 0)-1} \int_{t+\omega}^{t+2 \omega} \beta(s) y(s) e_{-\alpha}(s, t+\omega) \Delta s \\
& =\frac{1}{e_{-\alpha}(\omega, 0)-1} \int_{t}^{t+\omega} \beta(s+\omega) y(s+\omega) e_{-\alpha}(s+\omega, t+\omega) \Delta s \\
& =\frac{1}{e_{-\alpha}(\omega, 0)-1} \int_{t}^{t+\omega} \beta(s) y(s) e_{-\alpha}(s, t) \Delta s
\end{aligned}
$$

and

$$
\begin{aligned}
\Phi_{2} z(t+\omega) & =\frac{1}{1-e_{\ominus(-\alpha)}(\omega, 0)} \int_{t+\omega}^{t+2 \omega} \frac{e_{\ominus(-\alpha)}(s, t+\omega)}{1-\mu(s) \alpha(s)} \gamma(s) x(\sigma(s)) \Delta s \\
& =\frac{1}{1-e_{\ominus(-\alpha)}(\omega, 0)} \int_{t}^{t+\omega} \frac{e_{\ominus(-\alpha)}(s+\omega, t+\omega)}{1-\mu(s+\omega) \alpha(s+\omega)} \gamma(s+\omega) x(\sigma(s+\omega)) \Delta s \\
& =\frac{1}{1-e_{\ominus(-\alpha)}(\omega, 0)} \int_{t}^{t+\omega} \frac{e_{\ominus(-\alpha)}(s, t)}{1-\mu(s) \alpha(s)} \gamma(s) x(\sigma(s)) \Delta s,
\end{aligned}
$$

which means that $\Phi z \in \mathbb{X}$.

Next, we prove that $\Phi$ is a contraction mapping. Together with

$$
e_{-\alpha}(s, t)=e^{\int_{t}^{s} \xi_{\mu(\tau)}(-\alpha(\tau)) \Delta \tau} \leq e^{\int_{t}^{t+\omega}\left|\xi_{\mu(\tau)}(-\alpha(\tau))\right| \Delta \tau}=e^{\int_{0}^{\omega}\left|\xi_{\mu(\tau)}(-\alpha(\tau))\right| \Delta \tau}, \quad t \leq s \leq t+\omega, s \in \mathbb{T},
$$


for any $z_{1}(t)=\left(x_{1}(t), y_{1}(t)\right)^{T}, z_{2}(t)=\left(x_{2}(t), y_{2}(t)\right)^{T} \in \mathbb{X}$, we have

$$
\begin{aligned}
\left|\Phi_{1} z_{1}-\Phi_{1} z_{2}\right|_{0} & =\max _{t \in[0, \omega]_{\mathbb{T}}}\left|\frac{1}{e_{-\alpha}(\omega, 0)-1} \int_{t}^{t+\omega}\left(\beta(s) y_{1}(s) e_{-\alpha}(s, t)-\beta(s) y_{2}(s) e_{-\alpha}(s, t)\right) \Delta s\right| \\
& \leq \max _{t \in[0, \omega]_{\mathbb{T}}} \frac{1}{\left|e_{-\alpha}(\omega, 0)-1\right|} \int_{t}^{t+\omega} e_{-\alpha}(s, t)|\beta(s)|\left|y_{1}(s)-y_{2}(s)\right| \Delta s \\
& \leq \frac{\omega \beta^{+} e^{\int_{0}^{\omega}\left|\xi_{\mu(\tau)}(-\alpha(\tau))\right| \Delta \tau}}{\left|e_{-\alpha}(\omega, 0)-1\right|}\left|y_{1}-y_{2}\right|_{0}
\end{aligned}
$$

and

$$
\begin{aligned}
& \left|\Phi_{2} z_{1}-\Phi_{2} z_{2}\right|_{0} \\
& \quad=\max _{t \in[0, \omega]_{\mathbb{T}}}\left|\frac{1}{1-e_{\ominus(-\alpha)}(\omega, 0)} \int_{t}^{t+\omega} \frac{e_{\ominus(-\alpha)}(s, t)}{1-\mu(s) \alpha(s)} \gamma(s)\left(x_{1}(\sigma(s))-x_{2}(\sigma(s))\right) \Delta s\right| \\
& \quad \leq \max _{t \in[0, \omega]_{\mathbb{T}}} \frac{1}{\left|1-e_{\ominus(-\alpha)}(\omega, 0)\right|} \int_{t}^{t+\omega} \frac{e_{\ominus(-\alpha)}(s, t)}{|1-\mu(s) \alpha(s)|}|\gamma(s)|\left|x_{1}(\sigma(s))-x_{2}(\sigma(s))\right| \Delta s \\
& \quad \leq \frac{\omega \gamma^{+} e^{\int_{0}^{\omega}\left|\xi_{\mu(\tau)}(-\alpha(\tau))\right| \Delta \tau}\left|x_{1}-x_{2}\right|_{0} .}{\left|1-e_{\ominus(-\alpha)}(\omega, 0)\right| \min _{s \in[0, \omega]}\{|1-\mu(s) \alpha(s)|\}}
\end{aligned}
$$

Hence, we have

$$
\left\|\Phi z_{1}-\Phi z_{2}\right\| \leq \theta\left\|z_{1}-z_{2}\right\|<\left\|z_{1}-z_{2}\right\|
$$

It follows that $\Phi$ is a contraction. Therefore $\Phi$ has a fixed point in $\mathbb{X}$, that is, (1.1) has a unique periodic solution in $\mathbb{X}$. This completes the proof.

\section{Exponential stability of periodic solution}

In this section, we study the exponential stability of the periodic solution to (1.1).

Theorem 4.1 Assume that $\left(\mathrm{H}_{1}\right)$ and $\left(\mathrm{H}_{2}\right)$ hold. Suppose further that $\alpha^{-}>\gamma^{+}$. Then the periodic solution of (1.1) is exponentially stable.

Proof By Theorem 3.1, one can see that (1.1) has a unique $\omega$-periodic solution $z^{*}(t)=$ $\left(x^{*}(t), y^{*}(t)\right)^{T}$. Suppose that $z(t)=(x(t), y(t))^{T}$ is an arbitrary solution of (1.1). Denote $w(t)=(u(t), v(t))^{T}$, where $u(t)=x(t)-x^{*}(t), v(t)=y(t)-y^{*}(t)$. Then it follows from (1.1) that

$$
\left\{\begin{array}{l}
u^{\Delta}(t)=\alpha(t) u(\sigma(t))+\beta(t) v(t) \\
v^{\Delta}(t)=-\gamma(t) u(\sigma(t))-\alpha(t) v(t)
\end{array}\right.
$$

For $t_{0} \in \mathbb{T}$, by Theorem 2.74 and Theorem 2.77 in [8], we have

$$
u(t)=e_{\ominus(-\alpha)}\left(t, t_{0}\right) u\left(t_{0}\right)+\int_{t_{0}}^{t} e_{\ominus(-\alpha)}(t, s) \beta(s) v(s) \Delta s
$$

and

$$
\nu(t)=e_{-\alpha}\left(t, t_{0}\right) \nu\left(t_{0}\right)-\int_{t_{0}}^{t} e_{-\alpha}(t, \sigma(s)) \gamma(s) u(\sigma(s)) \Delta s .
$$


Take a constant $\lambda>0$ with $-\lambda \in \mathcal{R}^{+}$such that $\lambda>\beta^{+}(1+\vartheta \lambda)$ and $\alpha^{-} \epsilon_{1}>\gamma^{+}$, where $\epsilon_{1}=$ $\min _{t \in[0, \omega]_{\mathbb{T}}} e_{\ominus \lambda}\left(t, t_{0}\right)$. Let

$$
M>\max \left\{\frac{\lambda \epsilon_{2}}{\lambda-\beta^{+}(1+\vartheta \lambda)}, \frac{\alpha^{-}}{\alpha^{-} \epsilon_{1}-\gamma^{+}}\right\},
$$

where $\epsilon_{2}=\max _{t \in[0, \omega]_{\mathbb{T}}} e_{\lambda}\left(t, t_{0}\right)$. It is easy to verify that $M>1$ and hence, we have

$$
|w(t)| \leq\|w\|_{1}<M e_{\ominus \lambda}\left(t, t_{0}\right)\|w\|_{1}, \quad \forall t \in\left(-\infty, t_{0}\right]_{\mathbb{T}} .
$$

We claim that

$$
|w(t)|<M e_{\ominus \lambda}\left(t, t_{0}\right)\|w\|_{1}, \quad \forall t \in\left(t_{0},+\infty\right)_{\mathbb{T}},
$$

which means that

$$
|u(t)|<M e_{\ominus \lambda}\left(t, t_{0}\right)\|w\|_{1}, \quad \forall t \in\left(t_{0},+\infty\right)_{\mathbb{T}}
$$

and

$$
|v(t)|<M e_{\ominus \lambda}\left(t, t_{0}\right)\|w\|_{1}, \quad \forall t \in\left(t_{0},+\infty\right)_{\mathbb{T}} .
$$

By way of contradiction, assume that (4.4) does not hold, then we have the following three cases.

Case one: (4.6) is true and (4.5) is not true. Then there exists $t_{1} \in\left(t_{0},+\infty\right)_{\mathbb{T}}$ such that

$$
\left|u\left(t_{1}\right)\right| \geq M e_{\ominus \lambda}\left(t_{1}, t_{0}\right)\|w\|_{1}, \quad|u(t)|<M e_{\ominus \lambda}\left(t, t_{0}\right)\|w\|_{1}, \quad t \in\left(t_{0}, t_{1}\right)_{\mathbb{T}} .
$$

Hence, there must be a constant $p \geq 1$ such that

$$
\left|u\left(t_{1}\right)\right|=p M e_{\ominus \lambda}\left(t_{1}, t_{0}\right)\|w\|_{1}, \quad|u(t)|<M e_{\ominus \lambda}\left(t, t_{0}\right)\|w\|_{1}, \quad t \in\left(t_{0}, t_{1}\right)_{\mathbb{T}} .
$$

In view of (4.2), we have

$$
\begin{aligned}
\left|u\left(t_{1}\right)\right| & =\left|e_{\ominus(-\alpha)}\left(t_{1}, t_{0}\right) u\left(t_{0}\right)+\int_{t_{0}}^{t_{1}} e_{\ominus(-\alpha)}\left(t_{1}, s\right) \beta(s) v(s) \Delta s\right| \\
& \leq e_{\ominus(-\alpha)}\left(t_{1}, t_{0}\right)\left|u\left(t_{0}\right)\right|+\int_{t_{0}}^{t_{1}} e_{\ominus(-\alpha)}\left(t_{1}, s\right)|\beta(s)||v(s)| \Delta s \\
& \leq e_{\ominus(-\alpha)}\left(t_{1}, t_{0}\right)\|w\|_{1}+\beta^{+} p M\|w\|_{1} \int_{t_{0}}^{t_{1}} e_{\ominus(-\alpha)}\left(t_{1}, s\right) e_{\ominus \lambda}\left(s, t_{0}\right) \Delta s \\
& =e_{\ominus(-\alpha)}\left(t_{1}, t_{0}\right)\|w\|_{1}+\beta^{+} p M\|w\|_{1} e_{\ominus \lambda}\left(t_{1}, t_{0}\right) \int_{t_{0}}^{t_{1}} e_{-\alpha \ominus \lambda}\left(s, t_{1}\right) \Delta s \\
& =p M\|w\|_{1} e_{\ominus \lambda}\left(t_{1}, t_{0}\right)\left(\frac{1}{p M} e_{-\alpha \ominus \lambda}\left(t_{0}, t_{1}\right)+\beta^{+} \int_{t_{0}}^{t_{1}} e_{-\alpha \ominus \lambda}\left(s, t_{1}\right) \Delta s\right) \\
& \leq p M\|w\|_{1} e_{\ominus \lambda}\left(t_{1}, t_{0}\right)\left(\frac{1}{M} e_{\ominus \lambda}\left(t_{0}, t_{1}\right)+\beta^{+} \int_{t_{0}}^{t_{1}} e_{\ominus \lambda}\left(s, t_{1}\right) \Delta s\right)
\end{aligned}
$$




$$
\begin{aligned}
& =p M\|w\|_{1} e_{\ominus \lambda}\left(t_{1}, t_{0}\right)\left(\frac{1}{M} e_{\ominus \lambda}\left(t_{0}, t_{1}\right)+\frac{\beta^{+}}{\ominus \lambda} \int_{t_{0}}^{t_{1}} \ominus \lambda e_{\ominus \lambda}\left(s, t_{1}\right) \Delta s\right) \\
& \leq p M\|w\|_{1} e_{\ominus \lambda}\left(t_{1}, t_{0}\right)\left(\frac{1}{M} e_{\ominus \lambda}\left(t_{0}, t_{1}\right)+\frac{\beta^{+}}{\lambda}(1+\vartheta \lambda) e_{\ominus \lambda}\left(t_{0}, t_{1}\right)\right) \\
& <p M\|w\|_{1} e_{\ominus \lambda}\left(t_{1}, t_{0}\right),
\end{aligned}
$$

which is a contradiction.

Case two: (4.5) is true and (4.6) is not true. Then there exists $t_{2} \in\left(t_{0},+\infty\right)_{\mathbb{T}}$ such that

$$
\left|v\left(t_{2}\right)\right| \geq M e_{\ominus \lambda}\left(t_{2}, t_{0}\right)\|w\|_{1}, \quad|v(t)|<M e_{\ominus \lambda}\left(t, t_{0}\right)\|w\|_{1}, \quad t \in\left(t_{0}, t_{2}\right)_{\mathbb{T}} .
$$

In view of (4.3), we have

$$
\begin{aligned}
\left|v\left(t_{2}\right)\right| & =\left|e_{-\alpha}\left(t_{2}, t_{0}\right) v\left(t_{0}\right)-\int_{t_{0}}^{t_{2}} e_{-\alpha}\left(t_{2}, \sigma(s)\right) \gamma(s) u(\sigma(s)) \Delta s\right| \\
& \leq e_{-\alpha}\left(t_{2}, t_{0}\right)\left|v\left(t_{0}\right)\right|+\int_{t_{0}}^{t_{2}} e_{-\alpha}\left(t_{2}, \sigma(s)\right)|\gamma(s)||u(\sigma(s))| \Delta s \\
& \leq\|w\|_{1}+\gamma^{+} M\|w\|_{1} \int_{t_{0}}^{t_{2}} e_{-\alpha^{-}}\left(t_{2}, \sigma(s)\right) \Delta s \\
& =\|w\|_{1}+\frac{\gamma^{+} M\|w\|_{1}}{-\alpha^{-}} \int_{t_{0}}^{t_{2}}\left(-\alpha^{-}\right) e_{-\alpha}\left(t_{2}, \sigma(s)\right) \Delta s \\
& =\|w\|_{1}+\frac{\gamma^{+} M\|w\|_{1}}{-\alpha^{-}}\left(e_{-\alpha}\left(t_{2}, t_{0}\right)-1\right) \\
& \leq\|w\|_{1}+\frac{\gamma^{+} M\|w\|_{1}}{\alpha} \\
& =M\|w\|_{1} e_{\ominus \lambda}\left(t_{2}, t_{0}\right)\left(\frac{1}{M e_{\ominus \lambda}\left(t_{2}, t_{0}\right)}+\frac{\gamma^{+}}{\alpha e_{\ominus \lambda}\left(t_{2}, t_{0}\right)}\right) \\
& <M\|w\|_{1} e_{\ominus \lambda}\left(t_{2}, t_{0}\right),
\end{aligned}
$$

which is also a contradiction.

Case three: Both (4.5) and (4.6) are untrue. By case one and case two, we can obtain a contradiction. Therefore, (4.4) holds. Hence, we have that

$$
\left|z(t)-z^{*}(t)\right|_{1} \leq M\left\|z-z^{*}\right\|_{1} e_{\ominus \lambda}\left(t, t_{0}\right), \quad t \in \mathbb{T}, t \geq t_{0}
$$

which means that the periodic solution $z^{*}(t)$ of $(1.1)$ is exponentially stable. This completes the proof.

By Theorem 2.1 in [16], we have the following corollary.

Corollary 4.1 Assume that $\left(\mathrm{H}_{1}\right)$ and $\left(\mathrm{H}_{2}\right)$ hold. Suppose further that $\beta(t) \geq 0, \beta(t) \not \equiv 0$, $t \in[0, \omega]_{\mathbb{T}}$ and $\int_{0}^{\omega}\left(\gamma(t)-\frac{\alpha^{2}(t)}{\beta(t)}\right) \Delta t>0$. If

$$
\int_{0}^{\omega} \beta(t) \Delta t \int_{0}^{\omega} \gamma_{+}(t) \Delta t<4 \exp \left(-\int_{0}^{\omega}\left|\xi_{\mu(t)}(-\alpha(t))\right| \Delta t\right)
$$


or

$$
\int_{0}^{\omega}|\alpha(t)| \Delta t+\left(\int_{0}^{\omega} \beta(t) \Delta t\right)^{1 / 2}\left(\int_{0}^{\omega} \gamma_{+}(t) \Delta t\right)^{1 / 2}<2, \quad \gamma_{+}(t)=\max \{0, \gamma(t)\}
$$

Then (1.1) has a stable $\omega$-periodic solution.

\section{Examples}

In this section, we present two examples to illustrate the feasibility of our results obtained in previous sections.

Example 5.1 Consider the following Hamiltonian system on $\mathbb{T}=\mathbb{R}$ :

$$
\left\{\begin{array}{l}
x^{\prime}(t)=\alpha(t) x(t)+\beta(t) y(t), \\
y^{\prime}(t)=-\gamma(t) x(t)-\alpha(t) y(t), \quad t \in \mathbb{R}
\end{array}\right.
$$

in which we take the coefficients as follows:

$$
\alpha(t)=2+\sin t, \quad \beta(t)=0.03 \sin t, \quad \gamma(t)=0.05 \cos t .
$$

Since $\mathbb{T}=\mathbb{R}$, then $\mu(t)=0$. By calculating, we have

$$
\begin{aligned}
& \alpha^{+}=\bar{\alpha}=3, \quad \alpha^{-}=1, \quad \beta^{+}=0.03, \quad \gamma^{+}=0.05, \\
& \exp \left\{\int_{0}^{\omega}(-\alpha(s)) \mathrm{d} s\right\}=e^{-4 \pi}
\end{aligned}
$$

and $\theta \approx 0.134<1$. All the conditions in Theorem 3.1 and Theorem 4.1 are satisfied. Hence, (5.1) has an exponentially stable $2 \pi$-periodic solution.

Example 5.2 Consider the following Hamiltonian system on $\mathbb{T}=\mathbb{Z}$ :

$$
\left\{\begin{array}{l}
\Delta x(n)=\alpha(n) x(n+1)+\beta(n) y(n), \\
\Delta y(n)=-\gamma(n) x(n+1)-\alpha(n) y(n), \quad n \in \mathbb{Z},
\end{array}\right.
$$

in which we take the coefficients as follows:

$$
\alpha(n)=0.7+0.2 \sin \frac{n \pi}{3}, \quad \beta(n)=0.02 \cos \frac{n \pi}{3}, \quad \gamma(t)=0.04 \sin \frac{n \pi}{3} .
$$

Since $\mathbb{T}=\mathbb{Z}$, then $\mu(t)=1$. By calculating, we have $\alpha^{+}=\bar{\alpha}=0.9, \alpha^{-}=0.5, \beta^{+}=0.02, \gamma^{+}=$ 0.04 and

$$
\frac{\left|1-\prod_{k=1}^{\omega-1}(1-\alpha(k))\right| \exp \left\{\sum_{0}^{\omega-1} \log |1-\alpha(k)|\right\}}{\left|\prod_{k=1}^{\omega-1}(1-\alpha(k))\right|} \approx 1.147, \quad \theta \approx 0.1634<1 .
$$

All the conditions in Theorem 3.1 and Theorem 4.1 are satisfied. Hence, (5.2) has an exponentially stable 6-periodic solution. 
Remark 5.1 Since in (5.1) and (5.2), $\beta(t)$ or $\beta(n)$ may be negative, Theorem 2.1 in [16] is not suitable for our examples. But from our results, we can obtain that both (5.1) and (5.2) have exponentially stable $\omega$-periodic solutions.

\section{Competing interests}

The authors declare that they have no competing interests.

Authors' contributions

All authors contributed equally to the manuscript and typed, read and approved the final manuscript.

\section{Author details}

'Department of Mathematics, Yunnan University, Kunming, Yunnan 650091, People's Republic of China. ${ }^{2}$ School of Mathematics and Computer Science, Panzhihua University, Panzhihua, Sichuan 617000, People's Republic of China.

\section{Acknowledgements}

This study was supported by the National Natural Sciences Foundation of People's Republic of China under Grant 10971183.

Received: 6 January 2013 Accepted: 7 June 2013 Published: 25 June 2013

\section{References}

1. Ahlbrandt, C, Peterson, A: Discrete Hamiltonian Systems: Difference Equations, Continued Fractions, and Riccati Equations. Kluwer Academic, Boston (1996)

2. Tang, XH, Zhang, MR: Lyapunov inequalities and stability for linear Hamiltonian systems. J. Differ. Equ. 252, 358-381 (2012)

3. Liu, ZL, Su, JB, Wang, ZQ: A twist condition and periodic solutions of Hamiltonian systems. Adv. Math. 218, 1895-1913 (2008)

4. Han, ZQ: Computations of cohomology groups and nontrivial periodic solutions of Hamiltonian systems. J. Math Anal. Appl. 330(1), 259-275 (2007)

5. Sun, JT, Chen, HB, Nieto, JJ: Homoclinic solutions for a class of subquadratic second-order Hamiltonian systems. J. Math. Anal. Appl. 373, 20-29 (2011)

6. Hilger, S: Analysis on measure chains-a unified approach to continuous and discrete calculus. Results Math. 18, 18-56 (1990)

7. Bohner, M, Peterson, A: Dynamic Equations on Time Scales, an Introduction with Applications. Birkhäuser, Boston (2001)

8. Kaufmann, ER, Raffoul, YN: Periodic solutions for a neutral nonlinear dynamical equations on a time scale. J. Math. Anal. Appl. 319, 315-325 (2006)

9. Zhou, JW, Li, YK: Sobolev's spaces on time scales and its applications to a class of second order Hamiltonian systems on time scales. Nonlinear Anal. 73, 1375-1388 (2010)

10. Zhou, JW, Li, YK: Variational approach to a class of second order Hamiltonian systems on time scales. Acta Appl. Math. $117,47-69$ (2012)

11. Zhang, $H T$, Li, YK: Existence of positive periodic solutions for functional differential equations with impulse effects on time scales. Commun. Nonlinear Sci. Numer. Simul. 14, 19-26 (2009)

12. Li, YK, Yang, L, Wu, WQ: Anti-periodic solutions for a class of Cohen-Grossberg neural networks with time-varying delays on time scales. Int. J. Syst. Sci. 42, 1127-1132 (2011)

13. Li, YK, Wang, C: Uniformly almost periodic functions and almost periodic solutions to dynamic equations on time scales. Abstr. Appl. Anal. 2011, Article ID 341520 (2011)

14. Ahlbrandt, CD, Bohner, M: Hamiltonian systems on time scales. J. Math. Anal. Appl. 250, 561-578 (2000)

15. He, XF, Zhang, QM, Tang, XH: On inequalities of Lyapunov for linear Hamiltonian systems on time scales. J. Math. Anal. Appl. 381, 695-705 (2011)

16. Zafer, A: The stability of linear periodic Hamiltonian systems on time scales. Appl. Math. Lett. 26, 330-336 (2013)

17. Bohner, M, Zafer, A: Lyapunov type inequalities for planar linear dynamic Hamiltonian systems. Appl. Anal. Discrete Math. (2013). doi:10.2298/AADM130211004B

18. Adivar, M, Raffoul, YN: Existence of periodic solutions in totally nonlinear delay dynamic equations. Electron. J. Qual. Theory Differ. Equ. 1, 1-20 (2009)

doi:10.1186/1687-1847-2013-180

Cite this article as: Yang et al.: Existence and exponential stability of periodic solutions for a class of Hamiltonian systems on time scales. Advances in Difference Equations 2013 2013:180. 